\title{
A QUADRATURE METHOD FOR CONSTANT-COEFFICIENT CAUCHY SINGULAR INTEGRAL EQUATIONS ON AN INTERVAL
}

\author{
YOUNGMOK JEON ${ }^{1}$
}

(Received 29 September 1996; revised 12 February 1998)

\begin{abstract}
We consider a mesh grading quadrature method for real constant-coefficient Cauchy singular integral equations of index 0 . The quadrature method is based on the trapezoidal rule. A complete stability and convergence analysis is given by the use of the noncompact perturbation analysis as in Jeon [10] and Elschner and Stephan [7]. The order of convergence can be arbitrarily high if the order of mesh grading is high enough. We also provide an efficient way of evaluating asymptotics of the solution at the end points. Experimentally, we observe that the method also works well for Cauchy singular integral equations with variable coefficients.
\end{abstract}

\section{Cauchy singular integral equations}

We consider real constant-coefficient Cauchy singular integral equations

$$
a u(x)+\frac{b}{\pi} \text { p.v. } \int_{0}^{1} \frac{u(y)}{y-x} d y=f(x),
$$

where

$$
a^{2}+b^{2}=1, \quad a>0 .
$$

In the presence of a compact perturbation in (1.1), collectively compact operator analysis and perturbation analysis $[1,8]$, will allow our analysis to be extended to these cases. Cauchy singular integral equations have physical applications in elasticity and aerodynamics [14].

In this paper, we consider a natural airfoil solution of index 0 . We therefore set $u(x):=\rho(x) \phi(x)$, where

$$
\rho(x)=x^{\alpha}(1-x)^{\beta}
$$

'Department of Mathematics, Ajou University, Suwon 442-749, Korea.

(C) Australian Mathematical Society 2000, Serial-fee code 0334-2700/00 
and

$$
\alpha=\gamma, \quad \beta=-\gamma, \quad \text { where } \quad \gamma=\frac{1}{2 \pi i} \log \frac{a+b i}{a-b i},
$$

and we consider

$$
\mathscr{C} \phi(x):=(a I+b \mathscr{S}) \phi(x)=a \phi(x)+\frac{b}{\pi} \text { p.v. } \int_{0}^{l} \frac{\rho(y)}{\rho(x)} \frac{\phi(y)}{y-x} d y=\frac{f(x)}{\rho(x)} .
$$

Then $\mathscr{C}: L_{2}[0,1] \rightarrow L_{2}[0,1]$ is strongly elliptic and bijective [16]. Since $a>0$, it must be noted that $-1 / 2<\gamma<1 / 2$. If $f=\tilde{f} \rho \in H^{k}[0,1]$ with integer $k \geq 2$, then $\phi \in H^{k-|\gamma|}[0,1]\left[16\right.$, Corollary 6.38]. Here $H^{k}[0,1]$ denotes the usual Sobolev space of non-periodic functions on $[0,1]$, while $H^{k}$ denotes a Sobolev space of 1-periodic functions (see Section 4). From this point onwards, $C^{k}[0,1]$ and $C^{k}$ represent $k$-times differentiable non-periodic and periodic function spaces, respectively.

Instead of solving (1.3), we consider a mesh graded integral equation. Let us consider the well known mesh grading transformation [11], $w:[0,1] \rightarrow[0,1]$ such that

$$
w(t)=\frac{v^{q}(t)}{v^{q}(t)+v^{q}(1-t)}, \quad q \geq 2
$$

where

$$
v(t)=\left(\frac{1}{2}-\frac{1}{q}\right)(2 t-1)^{3}+\frac{1}{q}(2 t-1)+\frac{1}{2} .
$$

Then $w(t)$ satisfies

$$
w(t)=t^{q}+O\left(t^{q+1}\right), \quad 1-w(t)=(1-t)^{q}+O\left((1-t)^{q+1}\right)
$$

around 0 and 1 , respectively. The parameter $q$ is the order of mesh grading.

Multiplying (1.3) by $\sqrt{w^{\prime}(t)}$ and setting $\psi(t):=\phi(w(t)) \sqrt{w^{\prime}(t)}, r(t):=\rho(w(t))$ $=w^{\alpha}(t)(1-w(t))^{\beta}$ and $g(t):=f(w(t)) \sqrt{w^{\prime}(t) / r(t)}$, we have a transformed equation,

$$
\mathscr{C}^{q} \psi(t):=a \psi(t)+\frac{b}{\pi} \text { p.v. } \int_{0}^{1} \frac{r(s)}{r(t)} \frac{\sqrt{w^{\prime}(t)} \sqrt{w^{\prime}(s)}}{w(s)-w(t)} \psi(s) d s=g(t) .
$$

Since $\phi \rightarrow \psi$ is an isomorphism in $L_{2}[0,1]$, we have that

$$
\mathscr{C}^{q}: L_{2}[0,1] \rightarrow L_{2}[0,1] \text { is bijective. }
$$

We solve (1.5) for $\psi$ in preference to solving (1.3) for $\phi$. If $f$ in (1.1) is sufficiently smooth on $[0,1]$, then

$$
\psi(t)=t^{(q-1) / 2}(1-t)^{(q-1) / 2} h(t)
$$


with sufficiently smooth $h$ on $[0,1]$. Therefore, $\psi$ is considered to be a sufficiently smooth periodic function such that $\psi^{(j)}(0)=\psi^{(j)}(1)=0, \quad 0 \leq j \leq(q-1) / 2$ for $q$ large. We now suggest a numerical method based on the Fourier series approximation of the density function $\psi$ and the trapezoidal quadrature approximation with the subtraction of singularity for the singular integral operator. We therefore do not need to evaluate computationally costly Jacobi polynomials and this is what distinguishes our method from traditional polynomial methods $[5,9,20]$. In some cases polynomial methods may give rise to algorithms which converge more quickly than ours, but our method will be easier and more flexible to implement, especially in the presence of a compact perturbation.

In this paper we propose a modified version of the quadrature method introduced by Prössdorf and Rathsfeld in [15], in which they handle Cauchy singular integral equations of variable coefficients of index 0. They use the Simpson rule for the quadrature method, with which they achieve the convergence of $O\left(h^{\min \{4,(q-1) / 2\}}\right)$ for the approximation of the density function $\psi$ in (1.5). The evaluation of $\phi(0)$ and $\phi(1)$ in (1.3) is physically important, and they obtain the convergence of $O\left(h^{q(q-1) /(3 q-1)}\right)$ for $q \leq 8$. Moreover, they use the Toeplitz operator analysis for stability analysis that can be mathematically complicated.

In this paper, we use the trapezoidal rule for the quadrature instead of the Simpson rule. In applying a quadrature rule to the singular integral operator in (1.5), we use the subtraction of singularity by the periodic singular operator $\mathscr{H}$ (see (2.1)). This looks unconventional for the Cauchy singular operator on the interval $[0,1]$, but which has already been considered in [12]. Subsequent application of the perturbation theory in [10] and [7] yields a complete, concise stability analysis. Moreover, we obtain an optimal order of convergence, that is, $O\left(h^{q / 2}\right)$ for the density function $\psi$. For the evaluation of $\phi(0)$ and $\phi(1)$ we invent a new method, and the convergence of order $O\left(h^{q / 2-\lambda}\right)$, for an arbitrary $\lambda>0$, is achieved (in practice, $1 / 2 \leq \lambda \leq 1$ is desirable). As stated above, we theoretically obtain a convergence of arbitrarily high order with a high order mesh grading. From our observation, $q \leq 5$ looks fine for moderately large mesh points (see Section 7). So far, our analysis does not extend to variable coefficients, but numerical experiments suggest that our method is promising.

The paper is organized in the following way. In Section 2 we introduce a quadrature method for (1.5). In Section 3 strong ellipticity for the operator induced from $\mathscr{C}^{q}$ is obtained. Interpolatory projection and an induced solution projection on periodic function spaces are introduced in Section 4. Stability and convergence analysis are given in Sections 5 and 6. In Section 7 we present numerical results. Experimental numerical results for a variable coefficient case are also given and a good convergence is observed even though our analysis does not cover this case. 


\section{A quadrature method}

Here we introduce a quadrature method for $\mathscr{C}^{q}$. We write (1.5) in a symbolic form as

$$
\mathscr{C}^{a} \psi(t)=a \psi(t)+b \mathscr{H} \psi(t)+b \mathscr{K} \psi(t)=g(t),
$$

where

$$
\mathscr{H} \psi(t):=\text { p.v. } \int_{0}^{1} \cot (\pi(s-t)) \psi(s) d s
$$

and

$$
\mathscr{K} \psi(t):=\frac{1}{\pi} \int_{0}^{1}\left(\frac{r(s)}{r(t)} \frac{\sqrt{w^{\prime}(t)} \sqrt{w^{\prime}(s)}}{w(s)-w(t)}-\pi \cot (\pi(s-t))\right) \psi(s) d s .
$$

It must be noted that the kernel of $\mathscr{K}$ is smooth except for the four corners on $[0,1] \times[0,1]$, and it is extensible as an 1-periodic function on $\mathbb{R} \times \mathbb{R}$ except at integer pairs when the mesh grading is sufficiently large. Using the subtraction of singularity in the operator $\mathscr{H}$, then using the trapezoidal quadrature rule for the operators $\mathscr{H}$ and $\mathscr{K}$, we have an approximation operator $\mathscr{C}_{h}^{q}$ such that

$$
\begin{aligned}
\mathscr{C}_{h}^{q} \psi(t):= & \left.a \psi(t)+b \mid \mathscr{H}_{h} \psi(t)-\psi(t) \mathscr{H}_{h}(1)\right\}+b \mathscr{K}_{h} \psi(t) \\
= & a \psi(t)-b \psi(t)\left\{h \sum_{j=0}^{N-1} \cot (\pi(j h-t))\right\} \\
& +b \frac{h}{\pi} \sum_{j=0}^{N-1} \frac{r(j h)}{r(t)} \frac{\sqrt{w^{\prime}(t)} \sqrt{w^{\prime}(j h)}}{w(j h)-w(t)} \psi(j h),
\end{aligned}
$$

where with $N \in \mathbb{Z}$ and $h=1 / N$,

$$
\begin{aligned}
& \mathscr{H}_{h} \psi(t):=h \sum_{j=0}^{N-1} \cot (\pi(j h-t)) \psi(j h) \text { and } \\
& \mathscr{K}_{h} \psi(t):=\frac{h}{\pi} \sum_{j=0}^{N-1}\left(\frac{r(j h)}{r(t)} \frac{\sqrt{w^{\prime}(t)} \sqrt{w^{\prime}(j h)}}{w(j h)-w(t)}-\pi \cot (\pi(j h-t))\right) \psi(j h) .
\end{aligned}
$$

Collocation of (2.2) at nodes $\{k h\}_{k=0}^{N-1}$ cannot be defined, so we consider the mid-point collocation method.

We now introduce the $N$-dimensional trigonometric function space $T^{h}$ such that

$$
T^{h}:=\operatorname{span}\left\{e_{k} \mid e_{k}(t)=\exp (2 \pi i k t), N / 2<k \leq N / 2\right\}
$$


The periodic nature and symmetry of the cot function give us

$$
h \sum_{j=0}^{N-1} \cot \pi\left(j h-t_{k}\right)=0
$$

for $t_{k}=k h+h / 2[4,12]$. Our mid-point collocation method requires us to find $\psi_{h} \in T^{h}$ such that

$$
\mathscr{C}_{h}^{q} \psi_{h}\left(t_{k}\right)=a \psi_{h}\left(t_{k}\right)+b \frac{h}{\pi} \sum_{j=0}^{N-1} \frac{r(j h)}{r\left(t_{k}\right)} \frac{\sqrt{w^{\prime}\left(t_{k}\right)} \sqrt{w^{\prime}(j h)}}{w(j h)-w\left(t_{k}\right)} \psi_{h}(j h)=g\left(t_{k}\right),
$$

where $t_{k}=k h+h / 2,0 \leq k \leq N-1$. The Dirichlet kernel is then used to evaluate $\psi_{h}\left(t_{k}\right)$ :

$$
\psi_{h}(x)=h \sum_{j=0}^{N-1} E(x, j h) \psi_{h}(j h)
$$

where

$$
E(x, y)= \begin{cases}\cos (\pi(x-y)) \frac{\sin (N \pi(x-y))}{\sin (\pi(x-y))} & N: \text { even } \\ \frac{\sin (N \pi(x-y))}{\sin (\pi(x-y))} & N: \text { odd }\end{cases}
$$

But the stability of the above collocation method is unknown, and we need to modify the integral operator $\mathscr{K}_{h}$ around the end points to have a complete stability analysis. Our modified method requires us to find $\psi_{h} \in T^{h}$ such that

$$
\begin{aligned}
& a \psi_{h}\left(t_{k}\right)-b h \sum_{j=0}^{N-1} \cot \pi\left(j h-t_{k}\right) \psi_{h}(j h) \\
& \quad+b \frac{h}{\pi} \sum_{j=i^{*}}^{N-1-i^{*}}\left\{\frac{r(j h)}{r\left(t_{k}\right)} \frac{\sqrt{w^{\prime}\left(t_{k}\right)} \sqrt{w^{\prime}(j h)}}{w(j h)-w\left(t_{k}\right)}-\cot \left(j h-t_{k}\right)\right\} \psi_{h}(j h)=g\left(t_{k}\right), \\
& 0 \leq k \leq N-1 .
\end{aligned}
$$

Here the modification appears only for theoretical purposes, and we observe that $i^{*}=0$ is sufficient for stability in numerical experiments. We can rewrite (2.6) in symbolic form as

$$
\mathscr{C}_{h}^{q} \psi_{h}\left(t_{k}\right)=a \psi_{h}\left(t_{k}\right)+b \mathscr{H}_{h} \psi_{h}\left(t_{k}\right)+b \mathscr{K}_{h} T_{i^{*} h} \psi_{h}\left(t_{k}\right)=g\left(t_{k}\right), \quad 0 \leq k \leq N-1,
$$

where

$$
T_{r} \psi(x)= \begin{cases}\psi(x), & x \in[r, 1-r] \\ 0, & \text { otherwise }\end{cases}
$$

for $0 \leq r<1 / 2$. 


\section{Strong ellipticity}

The Hilbert operator $\mathscr{H}$ has well-known properties such as $\mathscr{H} 1=0$ and $\mathscr{H}^{2}=$ $\mathscr{J}-I[14]$. Then $\mathscr{H} \mathscr{J}=\mathscr{J} \mathscr{H}=0$ and simple calculation yields

$$
\left(a l-b \mathscr{H}+\frac{b^{2}}{a} \mathscr{J}\right)(a I+b \mathscr{H})=I,
$$

where $\mathscr{J} \phi(t)=\int_{0}^{1} \phi(s) d s$.

Multiplying both sides of $(2.1)$ by $(a I+b \mathscr{H})^{-1}$, we have

$$
(a I+b \mathscr{H})^{-1} \mathscr{C}^{q}=1+b \mathscr{L}+\mathscr{E}
$$

where

$$
\mathscr{L}:=(a l-b \mathscr{H}) \mathscr{K}
$$

and $\mathscr{E}:=b^{3} / a \mathscr{J} \mathscr{K}$ is a compact operator. Henceforth we shall assume that $\mathscr{E}$ is a generic compact operator which can be different on each appearance.

In this section we will show that the operator $(I+b \mathscr{L}+\mathscr{E})$ is strongly elliptic on $L_{2}[0,1]$. First, we will show by a localization technique that $I+b \mathscr{L}$ is a matrix of Mellin convolution operators with a compact perturbation.

Define a smooth cut off function $\chi$ such that $\chi=1$ on $[0, \delta]$ and $\operatorname{support}(\chi) \subset$ $[0,1 / 2)$. Let $\chi_{0}(x):=\chi(x)$ and $\chi_{1}(x):=\chi(1-x)$. Then

$$
\begin{aligned}
\cot (\pi(s-t))= & \chi_{0}(t) \chi_{0}(s) \frac{1}{\pi(s-t)}-\left.\chi_{1}(t) \chi_{1}(s) \frac{1}{\pi(\tilde{s}-\tilde{t})}\right|_{\tilde{s}=1-s, i=1-t} \\
& -\left.\chi_{0}(t) \chi_{1}(s) \frac{1}{\pi(\tilde{s}+t)}\right|_{s=1-s}+\left.\chi_{1}(t) \chi_{0}(s) \frac{1}{\pi(s+\tilde{t})}\right|_{i=1-t}+T_{1}(t, s)
\end{aligned}
$$

and

$$
\begin{aligned}
\frac{r(s)}{r(t)} \frac{\sqrt{w^{\prime}(t)} \sqrt{w^{\prime}(s)}}{w(s)-w(t)}= & \chi_{0}(s) \chi_{0}(t) \frac{s^{(q-1) / 2+\alpha q} t^{(q-1) / 2-\alpha q}}{s^{q}-t^{q}} \\
& -\chi_{1}(s) \chi_{1}(t) \frac{\tilde{s}^{(q-1) / 2+\beta q} \tilde{t}^{(q-1) / 2-\beta q}}{\tilde{s}^{q}-\tilde{t}^{q}}+T_{2}(t, s) .
\end{aligned}
$$

Here note that $\left(T_{1}(t, s)-T_{2}(t, s)\right)$ is a sufficiently smooth periodic function for large $q$.

Introducing the Mellin transform on $L_{2}[0, \infty)$, we have that

$$
\widehat{f}(z)=\int_{0}^{\infty} x^{z-1} f(x) d x, \quad z=\frac{1}{2}+i y, y \in \mathbb{R} .
$$


For details of the Mellin transform, see [6] and [19]. We now introduce three Mellin convolution operators on the half interval $[0, \infty)$ :

$$
\begin{aligned}
\mathscr{G} u(t) & =\frac{1}{\pi} \text { p.v. } \int_{0}^{\infty} G\left(\frac{t}{s}\right) \frac{u(s)}{s} d s, \\
\mathscr{R} u(t) & =\frac{1}{\pi} \int_{0}^{\infty} R\left(\frac{t}{s}\right) \frac{u(s)}{s} d s \quad \text { and } \\
\mathscr{G}_{\alpha}^{q} u(t) & =\frac{1}{\pi} \text { p.v. } \int_{0}^{\infty} G_{\alpha}^{q}\left(\frac{t}{s}\right) \frac{u(s)}{s} d s,
\end{aligned}
$$

where

$$
G(t)=\frac{1}{1-t}, \quad R(t)=\frac{1}{1+t} \quad \text { and } \quad G_{\alpha}^{q}(t)=\frac{t^{(q-1) / 2-\alpha q}}{1-t^{q}} .
$$

Then the corresponding symbols are

$$
\begin{aligned}
\widehat{\mathscr{G}}(z) & =\cot (\pi z), \\
\widehat{\mathscr{R}}(z) & =\frac{1}{\sin (\pi z)} \text { and } \\
\widehat{\mathscr{G}}_{\alpha}^{q}(z) & =-\tan \left(\pi\left(\frac{z-1 / 2}{q}-\alpha\right)\right),
\end{aligned}
$$

where $z=1 / 2+i y, y \in \mathbb{R}$. Note that the Mellin symbol for $\widehat{\mathscr{G}}_{\alpha}^{q}$ is defined only for $-1 / 2<\alpha<1 / 2$ and so our analysis does not cover the cases of index -1 and 1 .

In view of (3.2) and (3.3), the operators $\mathscr{K}$ in (2.1) and $\mathscr{H}$ can be written as

$$
\begin{aligned}
& \mathscr{K}=\Pi\left[\begin{array}{cc}
\mathscr{G}_{\alpha}^{q}-\mathscr{G} & \mathscr{R} \\
-\mathscr{R} & -\mathscr{G}_{\beta}^{q}+\mathscr{G}
\end{array}\right] \Pi^{T}+\left(\mathscr{T}_{2}-\mathscr{T}_{1}\right) \quad \text { and } \\
& \mathscr{H}=\Pi\left[\begin{array}{ll}
\mathscr{G} & -\mathscr{R} \\
\mathscr{R} & -\mathscr{G}
\end{array}\right] \Pi^{T}+\mathscr{T}_{1},
\end{aligned}
$$

where $\Pi:=\left(\chi_{0}, \chi_{1}\right)$ and $\left\{\chi_{i}\right\}_{i=0,1}$ are considered to be operators such that $\chi_{0} f(x):=$ $\chi_{0}(x) f(x)$ and $\chi_{1} f(x):=\chi_{1}(x) f(1-x)$. Here $\left(\mathscr{T}_{2}-\mathscr{T}_{1}\right)$ is compact, where $\mathscr{T}_{1}$ and $\mathscr{T}_{2}$ are operators with kernels $T_{1}(t, s)$ and $T_{2}(t, s)$ in (3.2) and (3.3), respectively. It is not difficult to see that $\mathscr{T}_{1} \mathscr{K}$ is compact and

$$
\left(I-\Pi^{T} \Pi\right)\left[\begin{array}{cc}
b\left(\mathscr{G}_{\alpha}^{q}-\mathscr{G}\right) & b \mathscr{R} \\
-b \mathscr{R} & -b\left(\mathscr{G}_{\beta}^{q}-\mathscr{G}\right)
\end{array}\right] \Pi^{T}
$$

is a vector of compact operators (see Appendix B [10]); then

$$
I+b \mathscr{L}=I+\Pi\left[\begin{array}{cc}
a I-b \mathscr{G} & b \mathscr{R} \\
-b \mathscr{R} & a I+b \mathscr{G}
\end{array}\right]\left[\begin{array}{cc}
b\left(\mathscr{G}_{\alpha}^{q}-\mathscr{G}\right) & b \mathscr{R} \\
-b \mathscr{R} & -b\left(\mathscr{G}_{\beta}^{q}-\mathscr{G}\right)
\end{array}\right] \Pi^{T}+\mathscr{E}
$$


where $\mathscr{E}$ is a compact operator. To show that $(I+b \mathscr{L})$ is strongly elliptic, we only need to show that the real part of the $2 \times 2$ complex matrix

$$
\left(I+\widehat{+b \mathscr{L}}_{\text {local }}\right)(z):=I+\left[\begin{array}{cc}
a-b \widehat{\mathscr{G}}(z) & b \widehat{\mathscr{R}}(z) \\
-b \widehat{\mathscr{R}}(z) & a+b \widehat{\mathscr{G}}(z)
\end{array}\right]\left[\begin{array}{cc}
b\left(\widehat{\mathscr{G}}_{\alpha}^{q}-\widehat{\mathscr{G}}\right)(z) & b \widehat{\mathscr{R}}(z) \\
-b \widehat{\mathscr{R}}(z) & -b\left(\widehat{\mathscr{G}}_{\beta}^{q}-\widehat{\mathscr{G}}\right)(z)
\end{array}\right],
$$

at $1 / 2+i y, y \in \mathbb{R}$, is positive definite uniformly in $y$. Using that

$$
I=\left[\begin{array}{cc}
a-b \widehat{\mathscr{G}}(z) & b \widehat{\mathscr{R}}(z) \\
-b \widehat{\mathscr{R}}(z) & a+b \widehat{\mathscr{G}}(z)
\end{array}\right]\left[\begin{array}{cc}
a+b \widehat{\mathscr{G}}(z) & -b \widehat{\mathscr{R}}(z) \\
b \widehat{\mathscr{R}}(z) & a-b \widehat{\mathscr{G}}(z)
\end{array}\right],
$$

simple calculation yields

$$
\begin{aligned}
\left(I+\widehat{\mathscr{L}}_{\text {local }}\right)(z)= & {\left[\begin{array}{cc}
a-b \cot (\pi z) & b / \sin (\pi z) \\
-b / \sin (\pi z) & a+b \cot (\pi z)
\end{array}\right] } \\
& \times\left[\begin{array}{cc}
a-b \tan \left(\pi\left(\frac{z-1 / 2}{q}-\alpha\right)\right) & 0 \\
0 & a+b \tan \left(\pi\left(\frac{z-1 / 2}{q}-\beta\right)\right)
\end{array}\right],
\end{aligned}
$$

where $z:=1 / 2+i y, y \in \mathbb{R}$.

Note that

$$
\cot (\pi z)=-i \tanh (\pi y), \quad \sin (\pi z)=\cosh (\pi y)
$$

and

$$
\begin{aligned}
\tan \pi\left(\frac{z-1 / 2}{q}-\alpha\right) & =\frac{1}{2} \frac{-\sin (2 \alpha \pi)+i \sinh (2 \pi y / q)}{\cosh ^{2}(\pi y / q)-\sin ^{2}(\alpha \pi)} \\
& :=u_{\alpha}(v)+i v_{\alpha}(v) .
\end{aligned}
$$

When $\alpha=-\beta$, we have $u_{\alpha}=-u_{\beta}$ and $v_{\alpha}=v_{\beta}$. Then simple calculation with $\alpha=-\beta=(1 / 2 \pi i) \log ((a+b i) /(a-b i))$ gives us

$$
\operatorname{Re}\left\langle\left(I \widehat{b}_{\text {local }}\right)(z) v, v\right\rangle \geq\langle L v, v\rangle,
$$

where

$$
L:=\left[\begin{array}{cc}
a\left(a-b u_{\alpha}\right)+b^{2} \tanh (\pi y) v_{\alpha} & 0 \\
0 & a\left(a-b u_{\alpha}\right)+b^{2} \tanh (\pi y) v_{\alpha}
\end{array}\right] .
$$

Here $\langle\cdot, \cdot\rangle$ is the usual inner product in $\mathbb{C}^{2}$. Using $\alpha \pi=\tan ^{-1}(b / a)$ and $a^{2}+b^{2}=1$ (see (1.1)),

$$
\begin{aligned}
a\left(a-b u_{\alpha}\right)+b^{2} \tanh (\pi y) v_{\alpha}= & a^{2}+\frac{a^{2} b^{2}}{\cosh ^{2}(\pi y / q)-b^{2}} \\
& +b^{2} \frac{\sinh (2 \pi y / q) \sinh (\pi y)}{\left(\cosh ^{2}(\pi y / q)-b^{2}\right) \cosh (\pi y)} \\
\geq & a^{2} .
\end{aligned}
$$


Then we have the Gårding inequality for the operator $(I+\mathscr{L}+\mathscr{E})$, that is,

$$
((I+\mathscr{L}+\mathscr{E}) \phi, \phi) \geq C(\phi, \phi)-\left(\mathscr{E}^{\prime} \phi, \phi\right)
$$

where $C>0, \phi \in L_{2}[0,1]$ and $\mathscr{E}^{\prime}$ is a compact operator.

\section{Projection operators}

Let $H^{l}$ be the Sobolev space of periodic functions on an interval $[0,1]$, and let $\|\cdot\|_{l}$ be the norm for $H^{l}$ such that

$$
\|f\|_{i}^{2}=\sum_{k \in \mathbb{Z}} \max \{1,|k|\}^{2 l}|\hat{f}(k)|^{2}<\infty
$$

where

$$
f(x)=\sum_{k \in \mathbb{Z}} \hat{f}(k) e_{k}
$$

with $e_{k}(t)=e^{2 \pi i k t}$. It is worthy of note that $H^{0}=L_{2}[0,1]$.

Let $P_{h}: H^{l}(l>1 / 2) \rightarrow T^{h}$ be an interpolatory projection such that $\left(P_{h} f\right)\left(t_{k}\right)=$ $f\left(t_{k}\right)$, where $t_{k}=k h+\xi h, 0 \leq \xi<1$. Then $P_{h}$ has the standard convergence property [18] that

$$
\left\|P_{h} f-f\right\|_{s} \leq C h^{t-s}\|f\|_{t}, \quad f \in H^{t},
$$

for $0 \leq s \leq t, t>1 / 2$. In this paper, we restrict our attention to the mid-point collocation, that is, $P_{h}$ with $\xi=1 / 2$.

Since $\mathscr{H}_{h} \psi_{h}-\psi_{h} \mathscr{H}_{h}(1)=\mathscr{H} \psi_{h}$ for $\psi_{h} \in T^{h}[10], P_{h} \mathscr{H}_{h}(1)=0$ (that is, (2.3)) and $T^{h}$ is invariant under $\mathscr{H}$, thus

$$
P_{h} \mathscr{H}_{h} \psi_{h}=P_{h} \mathscr{H} \psi_{h}=\mathscr{H} \psi_{h}
$$

Therefore, our modified collocation method (that is, (2.7)) can be rewritten in a symbolic form as

$$
a \psi_{h}+b \mathscr{H} \psi_{h}+b P_{h} \mathscr{K}_{h} T_{i^{\star} h} \psi_{h}=P_{h} g
$$

Consider a solution operator $R_{h}: H^{l}(L>1 / 2) \rightarrow T^{h}$ such that

$$
(a I+b \mathscr{H}) R_{h} \psi=P_{h}(a+b \mathscr{H}) \psi .
$$


Then $R_{h}=(a I+b \mathscr{H})^{-1} P_{h}(a I+b \mathscr{H})$, and it is easily checked that $R_{h}$ is a projection operator with the approximation property

$$
\left\|R_{h} \psi-\psi\right\|_{s} \leq C h^{t-s}\|\psi\|_{t}
$$

for $0 \leq s \leq t, t>1 / 2$. With $R_{h},(4.3)$ can be written as:

$$
\left(I+b R_{h} \mathscr{M}_{h} T_{i^{*} h}\right) \psi_{h}=R_{h} \tilde{g},
$$

where $\mathscr{M}_{h}=(a+b \mathscr{H})^{-1} \mathscr{K}_{h}$ and $\tilde{g}=(a+b \mathscr{H})^{-1} g$. Equation (2.1) may also be rewritten as

$$
(I+b \mathscr{M}) \psi=\tilde{g}
$$

with $\mathscr{M}=(a+b \mathscr{H})^{-1} \mathscr{K}$.

\section{Stability}

In this section we prove the stability of the operator $\left(I+b R_{h} \mathscr{M}_{h} T_{i^{*} h}\right): T^{h} \rightarrow T^{h}$ for a sufficiently large $i^{*}>0$ independent of $h$. As a first step, we introduce a stability result for a finite section operator.

THEOREM 5.1. For any $i^{*}>0$, there exists $h_{0}>0$ such that

$$
\left\|\left(I+b \mathscr{M} T_{i^{*} h}\right) \psi\right\|_{0} \geq C\|\psi\|_{0}, \quad \psi \in L_{2}[0,1],
$$

for all $0<h<h_{0}$ and $C$ independent of $h$.

Proof. Theorem 5.1 follows from the fact that $(I+b \mathscr{M})$ is strongly elliptic on $H^{0}$ (Section 3), $T_{i^{*} h}: H_{0} \rightarrow L_{2}\left[i^{*} h, 1-i^{*} h\right]$ is a bounded projection and from the bijectiveness of the operator $(I+b \mathscr{M})$ (from (1.6) and $\left.I+b \mathscr{M}=(a I+b \mathscr{H})^{-1} \mathscr{C}^{q}\right)$. For a detailed proof, see $[6,10]$.

Before proceeding to our stability analysis, we shall introduce some properties that are satisfied by the operator $\mathscr{K}$ (see (2.1)).

Consider an integer $m$ such that $m<q(1 / 2-|\gamma|)$ (see Section 1 for the definitions of $q$ and $\gamma$ ). Then

P1. $K_{t}=K(t, \cdot) \in H^{m}$ for $t \in(0,1)$ and $K_{s}=K(\cdot, s) \in H^{m}$ for $s \in(0,1)$;

P2. For $i, j \in \mathbb{Z}^{+} \cup\{0\}, u, v \in R^{+} \cup\{0\}$ such that $-(1 / 2-|\gamma|) q<i-u<$ $(1 / 2-|\gamma|) q$ and $i+j=u+v$,

$$
\mathscr{K}_{u, v, i, j} \psi(t)=\int_{0}^{1}[t(1-t)]^{u}[s(1-s)]^{v}\left|D_{t}^{i} D_{s}^{j} K(t, s)\right| \psi(s) d s
$$

is a bounded operator in $H^{0}$. 
Here $D_{t}^{i}:=\partial^{i} / \partial t^{i}$ and $D_{s}:=\partial^{i} / \partial s^{i}$. For a sketch of proofs for $P_{1}$ and $P_{2}$, see Appendix.

REMARK 1. For the proofs of Theorems 5.2, 5.3, 5.4 and 6.1, $(1 / 2-|\gamma|) q>m=1$ is sufficient.

For a stability analysis of the operator $\left(I+b R_{h} \mathscr{M}_{h} T_{i^{*} h}\right)$, we need some preliminary theorems 5.2, 5.3 and 5.4. It must be remarked that much of the proofs for these theorems are simple generalizations of those in [10] and [7].

LEMMA 5.2. For the operators $\mathscr{M}=(a I+b \mathscr{H})^{-1} \mathscr{X}$ and $\mathscr{M}_{h}=(a I+b \mathscr{H})^{-1} \mathscr{K}_{h}$, we have the following estimates. For $0 \leq l \leq m$,

(1)

$$
\left\|D^{l}\left(\mathscr{M}-\mathscr{M}_{h}\right) T_{i^{*} h} u\right\|_{0} \leq \frac{C}{i^{*}\left(i^{*} h\right)^{l}}\|u\|_{0}+\frac{C h}{\left(i^{*} h\right)^{l}}\|u\|_{1}, \quad u \in H^{1}
$$

(2)

$$
\left\|D^{l} \mathscr{M}_{h} T_{i^{*} h} u\right\|_{0} \leq \frac{C}{\left(i^{*} h\right)^{l}}\|u\|_{0}, \quad u \in T^{h}
$$

PROOF. Since $(a I+b \mathscr{H})^{-1}$ and the differential operator $D$ commute,

$$
\left\|D^{l}\left(\mathscr{M}-\mathscr{M}_{h}\right) T_{i \bullet h} u\right\|_{0}=C\left\|D^{l}\left(\mathscr{K}-\mathscr{K}_{h}\right) T_{i^{*} h} u\right\|_{0}
$$

Because

$$
\begin{aligned}
\left|D^{l}\left(\mathscr{K}-\mathscr{K}_{h}\right) T_{i^{*} h} u(t)\right| \leq & C h \int_{i^{*} h}^{1-i^{*} h}\left|D_{t}^{l} D_{s} K(t, s) u(s)\right| d s \\
& +C h \int_{i^{*} h}^{1-i^{*} h}\left|D_{l}^{l} K(t, s) D u(s)\right| d s \\
\leq & \frac{C h}{\left(i^{*} h\right)^{l+1}} \int_{0}^{1}\left|w_{l+1}(s) D_{t}^{l} D_{s} K(t, s) u(s)\right| d s \\
& +\frac{C h}{\left(i^{*} h\right)^{l}} \int_{0}^{1}\left|w_{l}(s) D_{t}^{l} K(t, s) D u(s)\right| d s,
\end{aligned}
$$

where $w_{l}(t)=[t(1-t)]^{l}$, we have the estimate (5.3).

Since

$$
\left\|D^{l} \mathscr{M} T_{i^{*} h} u\right\|_{0}=\left\|\int_{i^{*} h}^{1-i^{*} h} D_{t}^{l} K(t, s) u(s) d s\right\|_{0} \leq \frac{C}{\left(i^{*} h\right)^{l}}\|u\|_{0}
$$


and using (5.3),

$$
\left\|D^{l} \mathscr{M}_{h} T_{i^{*} h} u\right\|_{0} \leq \frac{C}{\left(i^{*} h\right)^{l}}\left(\|u\|_{0}+h\|u\|_{1}\right) .
$$

For $u \in T^{h}$, the inverse estimate, $h\|u\|_{1} \leq\|u\|_{0}$ yields (5.4) immediately.

For the rest of this paper, the operators $\mathscr{M}$ and $\mathscr{M}_{h}$ represent the operators defined in Lemma 5.2.

THEOREM 5.3. For arbitrary $\epsilon>0$ there exists $i^{*}>0$ such that for each $v \in T^{h}$ (1)

$$
\left\|\left(\mathscr{M}-\mathscr{M}_{h}\right) T_{i^{*} h} \mathscr{M}_{h} T_{i^{*} h} v\right\|_{0} \leq \epsilon\|v\|_{0} \quad \text { and }
$$

(2)

$$
\left\|\left(\mathscr{M}-\mathscr{M}_{h}\right) T_{i^{*} h}\left(I-R_{h}\right) \mathscr{M}_{h} T_{i^{*} h} v\right\|_{0} \leq \epsilon\|v\|_{0}
$$

PROOF. From Lemma 5.2 (1) with $l=0$ and $u=\mathscr{M}_{h} T_{i^{*} h} v$,

$$
\left\|\left(\mathscr{M}-\mathscr{M}_{h}\right) T_{i^{*} h} \mathscr{M}_{h} T_{i^{*} h} v\right\|_{0} \leq \frac{C}{i^{*}}\|u\|_{0}+C h\|u\|_{1} .
$$

Using the estimate Lemma 5.2 (2), we have

$$
\left\|\left(\mathscr{M}-\mathscr{M}_{h}\right) T_{i^{*} h} \mathscr{M}_{h} T_{i^{*} h} v\right\|_{0} \leq \frac{C}{i^{*}}\|v\|_{0}
$$

Taking $i^{*}$ large enough, we have (5.6).

Using Lemma $5.2(1)$ with $l=0$ and $u=\left(I-R_{h}\right) \mathscr{M}_{h} T_{i^{*} h} v$,

$$
\left\|\left(\mathscr{M}-\mathscr{M}_{h}\right) T_{i^{*} h}\left(I-R_{h}\right) \mathscr{M}_{h} T_{i^{*} h} v\right\|_{0} \leq \frac{C}{i^{*}}\|u\|_{0}+C h\|u\|_{1} .
$$

Now, using the error estimate of $R_{h}$ and Lemma 5.2 (2),

$$
\|u\|_{0} \leq C h\left\|\mathscr{M}_{h} T_{i^{*} h} v\right\|_{1} \leq C h \frac{\|v\|_{0}}{\left(i^{*} h\right)}
$$

and

$$
\|u\|_{1} \leq C\left\|\mathscr{M}_{h} T_{i^{* h}} v\right\|_{1} \leq C \frac{\|v\|_{0}}{i^{*} h}
$$

Then

$$
\left\|\left(\mathscr{M}-\mathscr{M}_{h}\right) T_{i^{*} h}\left(I-R_{h}\right) \mathscr{M}_{h} T_{i^{*} h} v\right\|_{0} \leq \frac{C}{i^{*}}\|v\|_{0} .
$$

Now the estimate (5.7) is immediate. 
Now we prove the stability of our approximation operator. The proof is obtained by simple application of the perturbation theory that in [1] and [3].

THEOREM 5.4. There exists $i^{*}>0$ such that

$$
\left\|\left(I+b R_{h} \mathscr{M}_{h} T_{i^{*} h}\right) \psi\right\|_{0} \geq C\|\psi\|_{0}, \quad \psi \in T^{h},
$$

for all $h<h_{0}$.

PROOF. Since $\left(I+b \mathscr{M} T_{i^{*} h}\right)$ is invertible from Theorem 5.1, define

$$
B_{h}:=I-b\left(I+b \mathscr{M} T_{i^{*} h}\right)^{-1} R_{h} \mathscr{M}_{h} T_{i^{*} h} .
$$

Then simple calculation yields

$$
B_{h}\left(I+b R_{h} \mathscr{M}_{h} T_{i^{*} h}\right)=I-S_{h},
$$

where

$$
S_{h}=b^{2}\left(I+b \mathscr{M} T_{i^{*} h}\right)^{-1}\left(R_{h} \mathscr{M}_{h}-\mathscr{M}\right) T_{i^{*} h} R_{h} \mathscr{M}_{h} T_{i^{*} h}
$$

We will now show that $\left\|S_{h} \psi\right\|_{0}<q\|\psi\|_{0}, q<1$ for sufficiently large $i^{*}$. We have that

$$
\left\|S_{h} \psi\right\|_{0} \leq C\left\|\left(R_{h} \mathscr{M}_{h}-\mathscr{M}\right) T_{i^{*} h} R_{h} \mathscr{M}_{h} T_{i^{*} h} \psi\right\|_{0}
$$

Now

$$
\begin{aligned}
\|\left(R_{h}\right. & \left.\mathscr{M}_{h}-\mathscr{M}\right) T_{i^{*} h} R_{h} \mathscr{M}_{h} T_{i^{*} h} \psi \|_{0} \\
\leq & \left\|\left(\mathscr{M}_{h}-\mathscr{M}\right) T_{i^{*} h} \mathscr{M}_{h} T_{i^{*} h} \psi\right\|_{0}+\left\|\left(I-R_{h}\right) \mathscr{M}_{h} T_{i^{*} h} \mathscr{M}_{h} T_{i^{*} h} \psi\right\|_{0} \\
& \quad+\left\|\left(\mathscr{M}_{h}-\mathscr{M}\right) T_{i^{*} h}\left(I-R_{h}\right) \mathscr{M}_{h} T_{i^{*} h} \psi\right\|_{0} \\
& \quad+\left\|\left(I-R_{h}\right) \mathscr{M}_{h} T_{i^{*} h}\left(I-R_{h}\right) \mathscr{M}_{h} T_{i^{*} h} \psi\right\|_{0} \\
& \leq P_{1}+P_{2}+P_{3}+P_{4} .
\end{aligned}
$$

The estimates for $P_{1}$ and $P_{3}$ are obtained from Theorem 5.3. Using the error estimate for $R_{h}$, the estimate (5.5) and Lemma $5.2(2)$, we have

$$
\begin{aligned}
P_{2} & \leq C h\left\|\mathscr{M}_{h} T_{i^{*} h} \mathscr{M}_{h} T_{i^{*} h} \psi\right\|_{1} \\
& \leq \frac{C h}{i^{*} h}\left\|\mathscr{M}_{h} T_{i^{*} h} \psi\right\|_{0}+\frac{C h^{2}}{i^{*} h}\left\|\mathscr{M}_{h} T_{i^{*} h} \psi\right\|_{1} \\
& \leq \frac{C}{i^{*}}\|\psi\|_{0}+\frac{C}{\left(i^{*}\right)^{2}}\|\psi\|_{0} .
\end{aligned}
$$


In a manner similar to that used above,

$$
\begin{aligned}
P_{4} & \leq C h\left\|\mathscr{M}_{h} T_{i^{*} h}\left(I-R_{h}\right) \mathscr{M}_{h} T_{i^{*} h} \psi\right\|_{1} \\
& \leq \frac{C h}{i^{*} h}\left\|\left(I-R_{h}\right) \mathscr{M}_{h} T_{i^{*} h} \psi\right\|_{0}+\frac{C h^{2}}{i^{*} h}\left\|\left(I-R_{h}\right) \mathscr{M}_{h} T_{i^{*} h} \psi\right\|_{1} \\
& \leq \frac{C h^{2}}{i^{*} h}\left\|\mathscr{M}_{h} T_{i^{*} h} \psi\right\|_{1}+\frac{C h^{2}}{i^{*} h}\left\|\mathscr{M}_{h} T_{i^{*} h} \psi\right\|_{1} \\
& \leq \frac{C}{\left(i^{*}\right)^{2}}\|\psi\|_{0} .
\end{aligned}
$$

Taking $i^{*}$ sufficiently large, we have $\left\|S_{h} \psi\right\|_{0}<q\|\psi\|_{0}, q<1$. Therefore

$$
\left(I+b R_{h} \mathscr{M}_{h} T_{i^{*} h}\right)^{-1}=\left(I-S_{h}\right)^{-1} B_{h},
$$

and it is uniformly bounded independently of $h$.

\section{Error analysis}

The convergence analysis heavily depends on the Euler-Maclaurin formula [2] for periodic functions, and we supply here a modified version of it. Assume $f^{(k)}(0)=$ $f^{(k)}(1)$ for $k=1, \ldots, p-1$, and that $f^{(p)}$ is integrable, then

$$
\left|\int_{0}^{1} f(x) d x-h \sum_{j=0}^{N-1} f(j h)\right| \leq C h^{p} \int_{0}^{1}\left|f^{(p)}(x)\right| d x .
$$

A generalized Euler-Maclaurin formula [13] may extend Theorem 6.1 to the case where $p \geq 1, p \in \mathbb{R}^{+}$.

Introduce a space of functions: for $p \in \mathbb{Z}^{+}$,

$$
S^{p}:=\left\{\psi \mid D^{j} \psi(x):=[x(1-x)]^{p-j} \psi_{j}(x), \quad \psi_{j} \in H^{0}, j=0, \ldots, p\right\},
$$

with the norm defined as

$$
\|\psi\|_{S^{p}}:=\sum_{j=0}^{p}\left\|\psi_{j}\right\|_{0}
$$

Simple calculation yields an embedding, $S^{p} \subset H^{p}$.

We now come to the main convergence result of the paper.

THEOREM 6.1. Suppose the mesh grading is of order $q>2 p$ for an integer $p \geq 1$. The solution of (1.5), $\psi$, belongs to $S^{p}$ if $f$ in (1.1) belongs to $H^{p+1}[0,1]$. Then the approximating solution $\psi_{h}$ of (2.6) satisfies the convergence property:

$$
\left\|\psi-\psi_{h}\right\|_{0} \leq C h^{p}\|\psi\|_{S^{p}}
$$


Proof. Suppose that $f \in H^{p+1}[0,1]$. Then $\phi$ in (1.3) is in $H^{p+1-|r|}[0,1]$ (see Section 1), and by Sobolev embedding, $\phi \in C^{p}[0,1]$. Then $\psi(t)=\phi(w(t)) \sqrt{w^{\prime}(t)}$ satisfies that $\psi(t)=[t(1-t)]^{p} h(t)$, where $h \in L_{2}[0,1]$ and $h \in C^{p}(0,1)$ if $(q-$ 1) $/ 2>p-1 / 2$. Then $\psi \in S^{p}$.

Instead of (1.5) and (2.6), we analyze the equivalent equations (4.6) and (4.7). Using (4.7) for the substitution of $\tilde{g}$ in (4.6),

$$
\left(I+b R_{h} \mathscr{M}_{h} T_{i^{*} h}\right) \psi_{h}=R_{h}(I+b \mathscr{M}) \psi
$$

Simple calculation yields

$$
\left(I+b R_{h} \mathscr{M}_{h} T_{i^{*} h}\right)\left(\psi_{h}-\psi_{N}\right)=-\left(\psi_{N}-R_{h} \psi\right)+b R_{h}\left(\mathscr{M} \psi-\mathscr{M}_{h} T_{i^{*} h} \psi_{N}\right),
$$

where $\psi_{N} \in T^{h}$ and $\psi_{N}(k h)=\psi(k h), k=0, \ldots, N-1$. By Theorem 5.4, we have

$$
\left\|\psi_{h}-\psi_{N}\right\|_{0} \leq C\left\|\psi_{N}-R_{h} \psi\right\|_{0}+C\left\|R_{h}\left(\mathscr{M} \psi-\mathscr{M}_{h} T_{i^{*} h} \psi_{N}\right)\right\|_{0}
$$

First, from Section 4

$$
\left\|\psi_{N}-R_{h} \psi\right\|_{0} \leq\left\|\psi_{N}-\psi\right\|_{0}+\left\|\psi-R_{h} \psi\right\|_{0} \leq C h^{p}\|\psi\|_{p}
$$

Since $\psi_{N}(k h)=\psi(k h)$, we have $\mathscr{M}_{h} T_{i^{*} h} \psi_{N}=\mathscr{M}_{h} T_{i^{*} h} \psi$. Now,

$$
\left\|R_{h}\left(\mathscr{M} \psi-\mathscr{M}_{h} T_{i^{*} h} \psi\right)\right\|_{0} \leq\left\|\left(\mathscr{M}-\mathscr{M}_{h} T_{i^{*} h}\right) \psi\right\|_{0}+C h\left\|\left(\mathscr{M}-\mathscr{M}_{h} T_{i^{*} h}\right) \psi\right\|_{1} .
$$

Then we have

$$
\left\|R_{h}\left(\mathscr{M} \psi-\mathscr{M}_{h} T_{i^{*} h} \psi_{N}\right)\right\|_{0} \leq C h^{p}\|\psi\|_{S^{p}}
$$

if we show that

$$
\left\|D^{j}\left(\mathscr{M} \psi-\mathscr{M}_{h} T_{i^{*} h}\right) \psi\right\|_{0} \leq C h^{p-j}\|\psi\|_{S^{p}}
$$

for $j=0,1$. Therefore, by (6.5) and (6.6), we have an estimate for (6.4):

$$
\left\|\psi_{N}-\psi_{h}\right\|_{0} \leq C h^{p}\|\psi\|_{S^{p}} .
$$

The triangle inequality yields

$$
\left\|\psi-\psi_{h}\right\|_{0} \leq\left\|\psi-\psi_{N}\right\|_{0}+\left\|\psi_{N}-\psi_{h}\right\|_{0} \leq C h^{p}\|\psi\|_{s^{p}},
$$

and our theorem is proved. 
Let us now prove the estimate (6.7). Since $\mathscr{M}=(a I+b \mathscr{H})^{-1} \mathscr{K}$ and the differential operator $D$ and $(a I+b \mathscr{H})^{-1}$ commute,

$$
\begin{aligned}
\left\|D^{j}\left(\mathscr{M} \psi-\mathscr{M}_{h} T_{i^{*} h}\right) \psi\right\|_{0} \leq & C\left\|D^{j}\left(\mathscr{K}-\mathscr{K}_{h}\right) \psi\right\|_{0}+C\left\|D^{j} \mathscr{K}\left(I-T_{i^{*} h}\right) \psi\right\|_{0} \\
& +C\left\|D^{j}\left(\mathscr{K}-\mathscr{K}_{h}\right)\left(I-T_{i^{*} h}\right) \psi\right\|_{0} \\
= & C P_{1}+C P_{2}+C P_{3} .
\end{aligned}
$$

For $P_{1}$, by the Euler-Maclaurin formula,

$$
\begin{aligned}
P_{1} & \leq C h^{p-j}\left\|\int_{0}^{1}\left|D_{t}^{j} D_{s}^{p-j}(K(t, s) \psi(s))\right| d s\right\|_{0} \\
& \leq C h^{p-j} \sum_{l=0}^{p-j}\left\|\int_{0}^{1}\left|D_{s}^{l} D_{t}^{j} K(t, s) D^{p-j-l} \psi(s)\right| d s\right\|_{0} \\
& \leq C h^{p-j} \sum_{l=0}^{p-j}\left\|\int_{0}^{1}\left|D_{s}^{l} D_{t}^{j} K(t, s)[s(1-s)]^{j+l} \psi_{p-j-l}(s)\right| d s\right\|_{0} \\
& \leq C h^{p-j}\|\psi\|_{s^{p}} .
\end{aligned}
$$

For $w_{l}(x)=[x(1-x)]^{l}$, since $D^{j}\left(\mathscr{K} w_{j}\right)\left(\right.$ that is, $\left.D^{j} \mathscr{K} w_{j}(\phi):=D^{j} \mathscr{K}\left(w_{j} \cdot \phi\right)\right)$ is bounded in $H^{0}$,

$$
P_{2} \leq\left\|\left(D^{j} \mathscr{K} w_{j}\right)\left(w_{-j}\left(I-T_{i^{*} h}\right) \psi\right)\right\|_{0} \leq C\left\|w_{-j}\left(I-T_{i^{\star} h}\right) \psi\right\|_{0} \leq C\left(i^{*} h\right)^{p-j}\|\psi\|_{S^{p}}
$$

Now,

$$
\begin{aligned}
P_{3} \leq & \left\|\int_{0}^{i^{*} h} D_{t}^{j} K(t, s) \psi(s) d s-h \sum_{k=0}^{i^{*}-1} D_{t}^{j} K(t, k h) \psi(k h)\right\|_{0} \\
& +\left\|\int_{1-i^{*} h}^{l} D_{t}^{j} K(t, s) \psi(s) d s-h \sum_{k=N-i^{*}}^{N} D_{t}^{j} K(t, k h) \psi(k h)\right\|_{0},
\end{aligned}
$$

and

$$
\begin{aligned}
& \left\|\int_{0}^{i^{* h}} D_{t}^{j} K(t, s) \psi(s) d s-h \sum_{k=0}^{i^{*}-1} D_{t}^{j} K(t, k h) \psi(k h)\right\|_{0} \\
& \quad \leq C h\left\|\int_{0}^{i^{*} h} D_{t}^{j} D_{s}(K(t, s) \psi(s)) d s\right\|_{0} \\
& \quad \leq C h \sum_{l=0}^{1}\left\|\int_{0}^{l} D_{t}^{j} D_{s}^{l} K(t, s) w_{j+l}(s) w_{-j-l}(s)\left(I-T_{i^{*} h}\right) D^{1-l} \psi(s) d s\right\|_{0} \\
& \quad \leq C h \sum_{l=0}^{1}\left\|w_{-j-l}\left(I-T_{i^{*} h}\right) D^{(1-l)} \psi\right\|_{0} \leq C h\left(i^{*} h\right)^{p-j-1}\|\psi\|_{S^{p}} \leq C h^{p-j}\|\psi\|_{S^{p}}
\end{aligned}
$$


We will have the same bound for

$$
\left\|\int_{1-i^{*} h}^{1} D_{t}^{j} K(t, s) \psi(s) d s-h \sum_{k=N-i^{*}}^{N} D_{t}^{j} K(t, k h) \psi(k h)\right\|_{0} .
$$

Then $P_{3} \leq C h^{p-j}\|\psi\|_{p}$. Adding up $P_{1}, P_{2}$ and $P_{3}$, we have the estimate (6.7).

Once Theorem 6.1 is proved, we can extend the convergence estimate to an arbitrary Sobolev space $H^{s}, 0<s \leq p$. Then we can even have a convergence result on the norm, $\|\cdot\|_{\infty}$, using the Sobolev embedding, $H^{s} \subset C^{0}, s>1 / 2$, where $C^{0}$ is a continuous periodic function space.

COROLLARY 6.2. Under the same assumptions as in Theorem 6.1 , for $0 \leq s \leq p$,

$$
\left\|\psi-\psi_{h}\right\|_{s} \leq C h^{p-s}\|\psi\|_{s p}
$$

PROOF. In the proof of Theorem 6.1 (see (6.7)), we have

$$
\left\|\psi_{N}-\psi_{h}\right\|_{0} \leq C h^{p}\|\psi\|_{S^{p}}
$$

where $\psi_{N} \in T^{h}$ and $\psi(k h)=\psi_{N}(k h),(k=0, \ldots, N-1)$. Then

$$
\left\|\psi_{N}-\psi_{h}\right\|_{s} \leq C h^{p-s}\|\psi\|_{S^{p}}
$$

by the inverse estimate on $T^{h}$. Since

$$
\left\|\psi-\psi_{N}\right\|_{s} \leq C h^{p-s}\|\psi\|_{p}
$$

we have the corollary.

\section{Numerical results}

Consider a Cauchy singular integral equation

$$
u(x)+\frac{1}{\pi} \text { p.v. } \int_{0}^{1} \frac{u(y)}{y-x} d y=\sqrt{2}
$$

Then (7.1) has a solution $u(x)=x^{1 / 4}(1-x)^{-1 / 4} \phi(x)$ with $\phi=1$. With our mesh graded equation (1.5), we will have a solution in the form, $\psi(t):=\phi(w(t)) \sqrt{w^{\prime}(t)}$. The second column of each table shows the convergence of $\psi_{h}$ of (2.4) to $\psi$ in $L_{2}$-norm, and the results show good concordance with Theorem 6.1 , that is,

$$
\left\|\psi-\psi_{h}\right\|_{0} \leq C h^{p}
$$


TABLE 1. Numerical results with $q=3$ and $n=\sqrt{N}$

\begin{tabular}{||c||c|r||c|r||}
\hline$h$ & $\left\|\psi-\psi_{h}\right\|_{0}$ & $r_{h}$ & $\left|\phi(1)-\phi_{n}^{h}(1)\right|$ & $r_{h}$ \\
\hline $1 / 16$ & $.109 \mathrm{e}-2$ & 1.53 & $.209 \mathrm{e}-1$ & 0.87 \\
\hline $1 / 32$ & $.396 \mathrm{e}-3$ & 1.46 & $.100 \mathrm{e}-1$ & 1.06 \\
\hline $1 / 64$ & $.143 \mathrm{e}-3$ & 1.47 & $.448 \mathrm{e}-2$ & 1.16 \\
\hline $1 / 128$ & $.509 \mathrm{e}-4$ & 1.49 & $.225 \mathrm{e}-2$ & .99 \\
\hline $1 / 256$ & $.181 \mathrm{e}-4$ & 1.49 & $.103 \mathrm{e}-2$ & 1.13 \\
\hline $1 / 512$ & $.642 \mathrm{e}-5$ & 1.50 & $.481 \mathrm{e}-3$ & 1.10 \\
\hline
\end{tabular}

TABLE 2. Numerical results with $q=5$ and $n=\sqrt{N}$

\begin{tabular}{||c||c|r||c|r||}
\hline$h$ & $\left\|\psi-\psi_{h}\right\|_{0}$ & $r_{h}$ & $\left|\phi(1)-\phi_{n}^{h}(1)\right|$ & $r_{h}$ \\
\hline $1 / 16$ & $.300 \mathrm{e}-3$ & 5.81 & $.351 \mathrm{e}-3$ & 6.07 \\
\hline $1 / 32$ & $.456 \mathrm{e}-5$ & 6.04 & $.208 \mathrm{e}-4$ & 4.08 \\
\hline $1 / 64$ & $.753 \mathrm{e}-6$ & 2.60 & $.623 \mathrm{e}-5$ & 1.74 \\
\hline $1 / 128$ & $.128 \mathrm{e}-6$ & 2.56 & $.181 \mathrm{e}-5$ & 1.78 \\
\hline $1 / 256$ & $.220 \mathrm{e}-7$ & 2.54 & $.420 \mathrm{e}-6$ & 2.11 \\
\hline $1 / 512$ & $.397 \mathrm{e}-8$ & 2.47 & $.743 \mathrm{e}-7$ & 2.50 \\
\hline
\end{tabular}

where $p$ equals the smallest integer less than $q / 2$, and $q$ is the order of mesh grading. In Tables 1, 2 and 3 we expect convergence of orders 1,2 and 2 respectively. But as mentioned in Section 6 we may have convergence of orders 1.5, 2.5 and 2.5 by the generalized Euler formula as in the tables.

We are also interested in evaluating the physically important constants $\phi(0)$ and $\phi(1)$. Here we describe a way of using $\psi_{h}$ to approximate $\phi(0)$ and $\phi(1)$.

Consider the Chebyshev polynomial on the interval $[0,1]$ : that is, $T_{n}(x)=\cos (n \theta)$ with $\theta=\cos ^{-1}(2 x-1)$. For any $\phi \in C[0,1]$,

$$
\phi(x)=\frac{C_{0}}{2}+\sum_{j=1}^{\infty} C_{j} T_{j}(x),
$$

where

$$
C_{j}=\frac{2}{\pi} \int_{0}^{1} \frac{\phi(x) T_{j}(x)}{x^{1 / 2}(1-x)^{1 / 2}} d x
$$

Let

$$
\phi_{n}(x):=\frac{C_{0}}{2}+\sum_{j=1}^{n} C_{j} T_{j}(x)
$$


TABLE 3. Numerical results with $q=5$ and $n=N$

\begin{tabular}{||c||c|r||c|r||}
\hline$h$ & $\left\|\psi^{2 h}-\psi^{h}\right\|_{0}$ & $r_{h}$ & $\phi_{n}^{h}(1)$ & $\phi_{n}^{h}(0)$ \\
\hline $1 / 16$ & $.493 \mathrm{e}-1$ & 2.57 & .8429641 & .0991423 \\
\hline $1 / 32$ & $.295 \mathrm{e}-2$ & 4.06 & .8342377 & .09314502 \\
\hline $1 / 64$ & $.251 \mathrm{e}-3$ & 3.55 & .8339479 & .0934511 \\
\hline $1 / 128$ & $.423 \mathrm{e}-4$ & 2.57 & .8338500 & .0935366 \\
\hline $1 / 256$ & $.730 \mathrm{e}-5$ & 2.53 & .8338204 & .0935660 \\
\hline $1 / 512$ & $.128 \mathrm{e}-5$ & 2.52 & .8338114 & .0935759 \\
\hline
\end{tabular}

Then by $[2,17]$,

$$
\left\|\phi-\phi_{n}\right\|_{\infty} \leq C n^{-r} \log (n), \quad \phi \in C^{r}[0,1]
$$

Using $T_{j}(1)=1$ and $T_{j}(0)=(-1)^{j}$,

$$
\begin{aligned}
& \phi(0)=\sum_{j=0}^{\infty}{ }^{\prime \prime}(-1)^{j} C_{j}, \\
& \phi(1)=\sum_{j=0}^{\infty} C_{j},
\end{aligned}
$$

where $\sum^{\prime \prime}$ is the summation obtained by halving the first term. Moreover,

$$
\begin{aligned}
\phi_{n}(1):=\sum_{j=0}^{n} C_{j} & =\frac{2}{\pi} \int_{0}^{1} \frac{\phi(x) F_{n}(x)}{x^{1 / 2}(1-x)^{1 / 2}} d x \\
& =\frac{2}{\pi} \int_{0}^{1} \frac{\psi(s) \sqrt{w^{\prime}(s)} F_{n}(w(s))}{w(s)^{1 / 2}(1-w(s))^{1 / 2}} d s,
\end{aligned}
$$

where

$$
F_{n}(x):=\sum_{j=0}^{n} T_{j}^{\prime \prime}(x)=\frac{1}{2}\left(\frac{e^{-i n \theta}-e^{i(n+1) \theta}}{1-e^{i \theta}}\right), \quad \theta=\cos ^{-1}(2 x-1) .
$$

Define

$$
\phi_{n}^{h}(1):=\frac{2}{\pi} \int_{0}^{1} \frac{\psi_{h}(s) \sqrt{w^{\prime}(s)} F_{n}(w(s))}{w(s)^{1 / 2}(1-w(s))^{1 / 2}} d s .
$$

Then $\phi_{n}^{h}(1)$ approximates $\phi(1)$, and the fourth column represents the numerical results. 
Now we estimate the convergence of $\phi_{n}^{h}(1)$ to $\phi(1)$.

$$
\begin{aligned}
\phi_{n}(1)-\phi_{n}^{h}(1)= & \int_{0}^{1} \frac{\left(\psi_{h}-\psi\right)(s) F_{n}(w(s)) \sqrt{w^{\prime}(s)}}{[w(s)(1-w(s))]^{1 / 2}} d s \\
= & \int_{0}^{h} \frac{\left(\psi_{h}-\psi\right)(s) F_{n}(w(s)) \sqrt{w^{\prime}(s)}}{[w(s)(1-w(s))]^{1 / 2}} d s \\
& +\int_{h}^{1-h} \frac{\left(\psi_{h}-\psi\right)(s) F_{n}(w(s)) \sqrt{w^{\prime}(s)}}{[w(s)(1-w(s))]^{1 / 2}} d s \\
& +\int_{1-h}^{1} \frac{\left(\psi_{h}-\psi\right)(s) F_{n}(w(s)) \sqrt{w^{\prime}(s)}}{[w(s)(1-w(s))]^{1 / 2}} d s \\
= & P_{1}+P_{2}+P_{3} .
\end{aligned}
$$

Then, since $\left|F_{n}(x)\right| \leq n$,

$$
\begin{aligned}
\left|P_{1}\right| & \leq n\left\|\psi_{h}-\psi\right\|_{\infty} \int_{0}^{h} \frac{\sqrt{w^{\prime}(s)}}{[w(s)(1-w(s))]^{1 / 2}[s(1-s)]^{1 / 2-\epsilon}}[s(1-s)]^{1 / 2-\epsilon} d s \\
& \leq C_{\epsilon} h^{1 / 2-\epsilon} n\left\|\psi_{h}-\psi\right\|_{\infty}
\end{aligned}
$$

for arbitrary $\epsilon>0$, and

$$
\begin{aligned}
\left|P_{2}\right| & \leq \int_{h}^{1-h} \frac{\left|\left(\psi_{n}-\psi\right)(s) F_{n}(w(s))\right| \sqrt{w^{\prime}(s)}}{[w(s)(1-w(s))]^{1 / 2}} d s \\
& \leq C n\left\|\psi_{h}-\psi\right\|_{0} \sqrt{\log (N)}
\end{aligned}
$$

since

$$
\int_{h}^{1-h} \frac{w^{\prime}(s)}{w(s)(1-w(s))} d s \leq C \log (N)
$$

and $h=1 / N$. We have the same estimate for $P_{3}$ as that for $P_{1}$. From the estimates (7.10) and (7.11) and using Corollary 6.2,

$$
\begin{aligned}
\left|\phi_{n}(1)-\phi_{n}^{h}(1)\right| & \leq C h^{1 / 2-\epsilon} n\left\|\psi_{h}-\psi\right\|_{\infty}+C n\left\|\psi_{h}-\psi\right\|_{0} \sqrt{\log (N)} \\
& \leq C n h^{p-\epsilon^{\prime}}\|\psi\|_{S^{p}}
\end{aligned}
$$

with $\epsilon^{\prime}>\epsilon$. Then (7.5) and (7.12) yield

$$
\left|\phi(1)-\phi_{n}^{h}(1)\right| \leq C h^{p-\lambda-\epsilon}
$$

for $n=N^{\lambda}, \phi \in C^{r}[0,1]$ with $\lambda(r+1) \geq p$ and arbitrary $\epsilon>0$. 
We will have the same kind of bound for $\left|\phi(0)-\phi_{n}^{h}(0)\right|$ by replacing $F_{n}$ in (7.7) and (7.8) with

$$
F_{n}:=\sum_{j=0}^{n}(-1)^{j} T_{j}(x)=\frac{1}{2}\left(\frac{e^{-i n \theta}+e^{i(n+1) \theta}}{1+e^{i \theta}}\right)
$$

with even $n$. In practice, optimizing $\lambda$ in (7.13) is an important issue, and $\lambda$ should be chosen appropriately according to the regularity of $\phi$. In our numerical test we have used $\lambda=1 / 2$ for Tables 1 and 2 and $\lambda=1$ for Table 3. Therefore, we expect convergence of orders 1,2 and 2 , respectively, and the fourth column of each table shows the convergence of $\phi_{n}^{h}(1)$ to $\phi(1)$. For the quadrature approximation for the integral in (7.8), we use the trapezoidal rule with 1024-points, while at points other than node points we evaluate $\psi_{h}$, using the interpolation formula (2.5).

Let us consider a variable coefficient singular integral equation,

$$
(1+\sin (\pi x)) u(x)+\left(-1+t^{2}(1-t)\right) \int_{0}^{1} \frac{1}{y-x} u(y) d y=\sin (x)
$$

the analysis of which is not contained in this paper. Then $u(x)=x^{-1 / 4}(1-x)^{1 / 4} \phi(x)$ [16], and Table 3 represents the approximate values of $\phi(0), \phi(1)$ and the convergence of $\psi_{h}$. We can see that the numbers are accurate up to 4 decimal digits with $N=128$, which shows a good convergence.

\section{Acknowledgements}

This research was undertaken when the author was visiting Universität Hannover, Hannover and WIAS, Berlin, Germany. He would like to express sincere thanks for the support of the above institutions. He also would like to sincerely thank Professor E. P. Stephan, Dr. A. Rathsfeld and Dr. J. Elschner for valuable suggestions in preparing the manuscript. The trip was also partly supported by the Applied Mathematics Research Center in KAIST. This research was supported by HAKJIN under the Grant number 01 D 0169.

\section{References}

[1] P. M. Anselone, Collectively Compact Operator Approximation Theory and Applications to Integral Equations (Prentice-Hall, Eaglewood Cliffs, 1971).

[2] K. E. Atkinson, An Introduction to Numerical Analysis, 2nd ed. (John Wiley Pub, New York, 1989). 
[3] K. E. Atkinson, A Survey of Numerical Methods for the Solution of Fredholm Integral Equations of the Second Kind (SIAM Pub., 1976), Chapter 2.1, Part II.

[4] S. M. Belotserkovski and I. K. Lifanov, Method of discrete vortices (CRC Press, Boca Raton, 1993).

[5] D. Elliott, "Convergence theorems for singular integral equations", in Numerical Solution of Integral Equations, Mathematical Concepts and Methods in Science and Engineering, 42 (ed. M.

A. Golberg), (Plenum Press, New York, 1990) 309-361.

[6] J. Elschner and I. G. Graham, "An optimal order collocation method for first kind boundary integral equations on polygons", Numer. Math. 70 (1995) 1-31.

[7] J. Elschner and E. P. Stephan, "A discrete collocation method for Symm's integral equation on curves with corners", submitted (1995).

[8] M. Golberg, "Perturbed projection methods for various classes of operator and integral equations", in Numerical Solution of Integral Equations. Mathematical Concepts and Methods in Science and Engineering, 42 (ed. M. A. Golberg) (Plenum Press, New York, 1990) 71-129.

[9] M. Golberg, "Introduction to the numerical solutions of Cauchy singular integral equations", in Numerical Solution of Integral Equations. Mathematical concepts and methods in science and engineering, 42 (ed. M. A. Golberg) (Plenum Press, New York, 1990) 183-308.

[10] Y. Jeon "A quadrature method for the Cauchy singular integral equations", J. Int. Eqns. Appl. 7, (1995) 425-461.

[11] R. Kress, "A Nyström method for boundary integral equations in domains with corners", Numer. Math. 58 (1990) 145-161.

[12] J. N. Lyness, "The Euler Maclaurin expansion for the Cauchy principal value integral", Numer. Math. 46 (1985) 611-622.

[13] J. N. Lyness and B. W. Ninham, "Numerical quadrature and asymptotic expansions", Math. Comp. 21 (1967) 162-178.

[14] N. I. Muskhelishvili, Singular Integral Equations (Dover Pub., 1992).

[15] S. Prössdorf and A. Rathsfeld, "Quadrature methods for strongly elliptic Cauchy singular integral equation on an interval", in Operator Theory: Advances and Applications, 41 (Birkhäuser Verlag, Basel, 1989) 435-471.

[16] S. Prössdorf and B. Silbermann, Numerical Analysis for Integral and Related Operator Equations (Birkhäuser, Basel, Boston, 1991).

[17] T. Rivlin, The Chebyshev Polynomials (Wiley, New York, 1974).

[18] J. Saranen and I. H. Sloan, "Quadrature methods for logarithmic-kernel equations on closed curves", IMA J. Numer. Anal. 12 (1992) 537-567.

[19] I. N. Sneddon, The Use of Integral Transforms, (McGraw-Hill, New York, 1972).

[20] E. Venturino, "Unconventional solution of singular integral equation", J. Int. Eqns. Appl. 4 (1992) $574-587$.

\section{A. Appendix}

Consider a class of functions $\mathscr{X}^{l, m, k+\alpha}$ for $l, m, k \in \mathbb{Z}^{+}$and $0<\alpha<1$. The function $K \in \mathscr{X}^{l, m, k+\alpha}$ if $K$ satisfies

$$
K(x)=\frac{Q(x)}{P(x)}+x^{k+\alpha} \frac{R(x)}{P(x)}
$$


where

$$
\begin{array}{ll}
P(x)=c_{0}+c_{1} x+\cdots+c_{n} x^{n}, & c_{0} \neq 0, c_{n} \neq 0, \\
Q(x)=d_{0}+d_{1} x+\cdots+d_{n-l} x^{n-l}, & d_{n-l} \neq 0, \\
R(x)=e_{0}+e_{1} x+\cdots+e_{n-m} x^{n-m}, & e_{0} \neq 0, e_{n-m} \neq 0 .
\end{array}
$$

LEMMA A.1. If $K \in \mathscr{X}^{l, m, k+\alpha}$, then

(1) $K^{\prime} \in \mathscr{X}^{l+1, m, k+\alpha-1}$;

(2) $x K^{\prime}(x)+l K(x) \in \mathscr{X}^{\mu, m, k+\alpha}, \mu \geq l+1$.

ProOF. (1) Simple calculation yields

$$
\begin{aligned}
K^{\prime}(x)= & \frac{Q^{\prime}(x) P(x)-Q(x) P^{\prime}(x)}{[P(x)]^{2}} \\
& +x^{k+\alpha-1} \frac{(k+\alpha) R(x) P(x)+x R^{\prime}(x) P(x)-x R(x) P^{\prime}(x)}{[P(x)]^{2}} .
\end{aligned}
$$

For $\left(Q^{\prime}(x) P(x)-Q(x) P^{\prime}(x)\right) /[P(x)]^{2}$, looking at the coefficient of $x^{2 n-l-1}$ in the numerator, we have $(n-l) d_{n-l} c_{n}-d_{n-l} n c_{n}=-l d_{n-l} c_{n} \neq 0$.

For $\left((k+\alpha) R(x) P(x)+x R^{\prime}(x) P(x)-x R(x) P^{\prime}(x)\right) /[P(x)]^{2}$, looking at the constant term in the numerator, $(k+\alpha) e_{0} c_{0} \neq 0$. Looking at the coefficient of $x^{2 n-m}$ in the numerator, $(k+\alpha) e_{n-m} c_{n}+(n-m) e_{n-m} c_{n}-e_{n-m} n c_{n}=(k+\alpha-m) e_{n-m} c_{n} \neq 0$. Then $K^{\prime} \in \mathscr{X}^{l+1, m, k+\alpha-1}$.

(2) Simple calculation yields

$$
\begin{aligned}
x K^{\prime}(x)+l K(x)= & \frac{x Q^{\prime}(x) P(x)-x Q(x) P^{\prime}(x)+l Q(x) P(x)}{[P(x)]^{2}} \\
& +x^{k+\alpha} \frac{(k+\alpha+l) R(x) P(x)+x R^{\prime}(x) P(x)-x R(x) P^{\prime}(x)}{[P(x)]^{2}} .
\end{aligned}
$$

Looking at the numerator of $\left(x Q^{\prime}(x) P(x)-x Q(x) P^{\prime}(x)+l Q(x) P(x)\right) /[P(x)]^{2}$, the coefficient of $x^{2 n-l}$ is $(n-l) d_{n-l} c_{n}-n d_{n-l} c_{n}+l d_{n-l} c_{n}=0$. Then the numerator is a polynomial of degree $\leq(2 n-l-1)$.

For $\left((k+\alpha+l) R(x) P(x)+x R^{\prime}(x) P(x)-x R(x) P^{\prime}(x)\right) /\left([P(x)]^{2}\right)$, as in the proof of (1), the constant term and the coefficient of $x^{2 n-m}$ are nonzero. Now the lemma is proved.

COROLlary A.2. For $K \in \mathscr{X}^{l, m, k+\alpha}$ and $i+j=u+v$,

$$
t^{u} s^{v} D_{t}^{i} D_{s}^{j}\left[K\left(\frac{t}{s}\right) \frac{1}{s}\right]=\tilde{K}\left(\frac{t}{s}\right) \frac{1}{s}
$$

where $\tilde{K} \in \mathscr{X}^{\mu, m, k+\alpha-i+u}, \mu \geq l+i+j-u$. 
PROOF. Note that

$$
D_{t}\left[K\left(\frac{t}{s}\right) \frac{1}{s^{\lambda}}\right]=K^{\prime}\left(\frac{t}{s}\right) \frac{1}{s^{\lambda+1}}
$$

and

$$
D_{s}\left[K\left(\frac{t}{s}\right) \frac{1}{s^{\lambda}}\right]=-\left[K^{\prime}\left(\frac{t}{s}\right) \frac{t}{s}+\lambda K\left(\frac{t}{s}\right)\right] \frac{1}{s^{\lambda+1}}
$$

Using Lemma A.1, it is clear that

$$
D_{i}^{i} D_{s}^{j}\left[K\left(\frac{t}{s}\right) \frac{1}{s}\right]=D_{s}^{j}\left[K^{(i)}\left(\frac{t}{s}\right) \frac{1}{s^{i+1}}\right]=L\left(\frac{t}{s}\right) \frac{1}{s^{1+i+j}},
$$

where $L \in \mathscr{X}^{\mu, m, k+\alpha-i}, K^{(i)} \in \mathscr{X}^{l+i, m, k+\alpha-i}, \mu \geq l+i+j$.

Then

$$
t^{u} s^{v} D_{t}^{i} D_{s}^{j}\left[K\left(\frac{t}{s}\right) \frac{1}{s}\right]=\tilde{K}\left(\frac{t}{s}\right) \frac{1}{s}
$$

where $\tilde{K}(x)=x^{u} L(x) \in \mathscr{X}^{\mu, m, k+\alpha-i+u}, \mu \geq l+i+j-u$.

Now let us look at the operator $\mathscr{K}$ in (2.1). We will show that $\mathscr{K}$ satisfies the properties (P1) and (P2) in Section 5. We have that

$$
\mathscr{K} \psi(t)=\frac{1}{\pi} \int_{0}^{1}\left(\frac{r(s)}{r(t)} \frac{\sqrt{w^{\prime}(t)} \sqrt{w^{\prime}(s)}}{w(s)-w(t)}-\pi \cot (\pi(s-t))\right) \psi(s) d s .
$$

Decompose the kernel $K(t, s)$ of $\mathscr{K}$ as follows.

$$
\begin{aligned}
K(t, s)= & \chi_{0}(t)\left[\frac{s^{(q-1) / 2+\alpha q} t^{(q-1) / 2-\alpha q}}{\pi\left(s^{q}-t^{q}\right)}-\frac{1}{\pi(s-t)}\right] \chi_{0}(s) \\
& -\chi_{0}(t) \chi_{1}(s) \frac{1}{\pi\{(1-s)+t\}}+\chi_{1}(t) \chi_{0}(s) \frac{1}{\pi\{s+(1-t)\}} \\
& -\chi_{1}(s) \chi_{1}(t)\left[\frac{(1-s)^{(q-1) / 2+\beta q}(1-t)^{(q-1) / 2-\beta q}}{\pi\left\{(1-s)^{q}-(1-t)^{q}\right\}}-\frac{1}{\pi\{(1-s)-(1-t)\}}\right] \\
& +E(t, s)
\end{aligned}
$$

where $E(t, s)$ is a continuous periodic function for $q>1$.

Looking at $K(t, s)$, it is clear that $K_{t}:=K(t, \cdot) \in H^{m}$ and $K_{s}:=K(\cdot, s) \in H^{m}$, and thus we have (P1). Recall that $m$ is an integer less than $q(1 / 2-|\gamma|)$, where $|\gamma| \geq \alpha$. 
According to the above decomposition of the kernel, we may decompose the operator $\mathscr{K}$ by

$$
\mathscr{K} \psi=\chi_{0} \mathscr{K}_{0,0} \chi_{0} \psi+\chi_{0} \mathscr{K}_{0,1} \chi_{1} \psi+\chi_{1} \mathscr{K}_{1,0} \chi_{0} \psi+\chi_{1} \mathscr{K}_{1,1} \chi_{1} \psi+\mathscr{E} \psi
$$

For simplicity, let us look only at $\mathscr{K}_{0,0}$. We have that

$$
\mathscr{K}_{0,0} \psi=\int_{0}^{\infty} K_{0,0}\left(\frac{t}{s}\right) \frac{1}{s} \psi(s) d s
$$

with

$$
K_{0,0}(x)=\frac{x^{(1 / 2-\alpha) q-1 / 2}-\left(x^{q-1}+\cdots+x+1\right)}{1-x^{q}},
$$

where $x=1$ is a removable singularity. Then $K_{0,0} \in \mathscr{X}^{1 . q,(1 / 2-\alpha) q-1 / 2}$. By Corollary A.2, since $K_{0,0} \in \mathscr{X}^{1, q,(1 / 2-\alpha) q-1 / 2}$,

$$
t^{u} s^{v} D_{t}^{i} D_{s}^{j} K_{0,0}(t, s) \frac{1}{s}=\tilde{K}\left(\frac{t}{s}\right) \frac{1}{s},
$$

where $\tilde{K} \in \mathscr{X}^{1+i+j-u, q .(1 / 2-\alpha) q-1 / 2-i+u}$. To have $x^{-1 / 2} \tilde{K}(x) \in L_{1}[0, \infty)$, we need that $(1 / 2-\alpha) q-1 / 2-i+u>-1 / 2$ and $(1 / 2-\alpha) q-1 / 2-i+u<q-1 / 2$ since $1+i+j-u \geq 1$. Therefore, if $-(1 / 2+\alpha) q<i-u<(1 / 2-\alpha) q$,

$$
\int_{0}^{\infty} t^{u} s^{v} D_{t}^{i} D_{s}^{j} K_{0,0}(t, s) \frac{1}{s} \phi(s)
$$

is a bounded operator on $L_{2}[0, \infty)$. 